above $38 \circ \mathrm{C}$, erythrocyte sedimentation rate over $30 \mathrm{~mm} / \mathrm{h}$ and monoarthralgia were defined as minor diagnostic criteria in middle and high risk populations. The aim of this study is to determine the effect of new diagnostic criterias on our clinical practice.

Methods Among a total of 1132 patients hospitalized in our Pediatric Cardiology Clinic between 2013 and 2019, data of 58 patients were diagnosed with ARF were investigated and the 3-year period before and after the updated Jones criteria were compared.

Results Fifteen of the 493 patients hospitalized between 2013-2015, 43 of the 639 patients hospitalized between 2016-2019 were diagnosed as ARF. There was a significant increase in the number of patients with ARF during the updated diagnostic criteria $(\mathrm{p}=0.007)$. Joint complaints were in the foreground in both periods and there was no difference between the distribution of patients who were evaluated as clinical and subclinical carditis $(p>0.05)$. In the previous period, there was no patient in whom monoarthritis and polyartralgia were accepted as major diagnostic criteria but, in the last three years, of the 17 patients, 8 had monoarthritis and 9 had polyarthralgia as the major diagnostic criteria. Of the ARF patients with polyartalgia defined as the major diagnostic criteria, 6 patient underwent diagnostic changes; 2 had juvenile idiopathic arthritis, 3 had familial Mediterranean fever and 1 had systemic lupus erythematosus. In all of these patients with high ASO levels, except for two patients with subclinical carditis, the diagnosis of ARF was made by polyartralgia, fever and high acute phase reactants. In a patient with carditis findings besides polyartalgia, the association of ARF and Takayasu arteritis was defined at the 8 th month of follow-up.

Conclusion With the updated Jones criteria, the number of patients diagnosed with ARF are increased. However, in the follow-up, some patients diagnoses were changed as collagen tissue diseases and vasculitis. New criterias may lead to diagnosis of some cases of ARF that may be overlooked, but may also lead to over-diagnosis. Therefore, collagen tissue diseases and vasculitis should be considered in the differential diagnosis of patients with polyartalgia, especially in patients with atypical joint involvement without carditis.

\section{GP30 ASSOCIATION OF ACE GENE INSERTION/DELETION POLYMORPHISMS WITH HYPERTENSION IN CHILDREN WITH GLOMERULONEPHRITIS}

Lyubov Rychkova*, Elena Belyaeva, Tatyana Bairova, Lyubov Kolesnikova. Scientific Centre for Family Health and Human Reproduction Problems, Irkutsk, Russian Federation

10.1136/archdischild-2019-epa.96

Background Kidney diseases are a common cause of secondary hypertension in children, which determines an unfavourable prognosis of the underlying disease. The secondary hypertension is a multifactorial complex disorder caused both by genetic and environmental factors. According to estimations approximately in $30 \%$ of cases, the interindividual variability in blood pressure is genetically determined. Numerous studies focused on the role of genetic variation in genes implicated in the renin-angiotensin system (RAS), particularly the angiotensin-converting enzyme (ACE) gene. The purpose of our study was designed to explore association of angiotensin converting enzyme (ACE) gene insertion/deletion (I/D) polymorphism with secondary hypertension in a group of children with glomerulonephritis.

Methods The study included two groups of children with glomerulonephritis, one of them - hypertensives (cases) and another group - normotensives (controls). Group with hypertension comprised 35 children, age $10.46 \pm 2.93$ years (20 males and 15 females), group without hypertension included 26 children, age $9.61 \pm 3.18$ years (18 males and 8 females). The variant genotype of ACE gene (I/D polymorphism; rs4646994) was determined using polymerase chain reaction (PCR). DNA was extracted from blood samples. DNA fragments length analysis after amplification and restriction was carried out in 7\% acrylamide gel. Statistical analysis was performed using STATISTICA, version 10.0 StatSoft Inc, USA.

Results We determine the distribution of genotype and allele frequencies in the hypertensives and normotensives groups of children with glomerulonephritis. The analysis showed that the frequencies of genotype II, ID, DD of ACE gene in the group of hypertensives were $14.3 \%, 25.7 \%, 60 \%$, in the group of normotensives were $76.9 \%, 15.4 \%, 7.7 \%$. We found statistically significant higher frequency of DD genotype $\left(\chi^{2}=8.44 ;\right.$ d.f. $\left.=1 ; p=0.004\right)$ and $D$ allele $(z=6.1$; $\mathrm{p}=0.001)$ in the hypertensives group in comparison with the normotensives group of children. We calculated the probability of DD genotype detection in the group of patients with glomerulonephritis by the odds ratio (OR) was calculated with 95\% confidence interval (95\% CI). Patients with the DD genotype had a higher risk of hypertensives than patients with the II, ID genotypes (OR: $18 ; 95 \% \mathrm{Cl}$ : 3.66 - 88.55).

Conclusions The findings revealed significant influence of $\mathrm{I} / \mathrm{D}$ polymorphism of ACE gene on hypertension in the groups of children with glomerulonephritis. There are significant associations between DD genotypes and secondary hypertension in children with glomerulonephritis.

\section{GP31 ENDOTHELIAL DYSFUNCTION AS THE MAIN COMPONENT OF ARTERIAL HYPERTENSION IN THE CHILDREN}

Olesya Horlenko*, Vasyl Rusyn, Natalia Sochka, Olena Debretseny, Fedir Horlenko, Galina Radovetska. Uzhhorod National University, Uzhhorod, Ukraine

\subsection{6/archdischild-2019-epa.97}

Backgruond and aim First of all, endothelial dysfunction (ED) have an importante role in modulation of cardiovascular risk in healthy individuals and patients with arterial hypertension. It is already known that the formation of hypertension is associated not only with the state of vascular tone, but also associated with a violation of the relaxation properties of the endothelium.

Methods We selected 80 adolescents aged 15-17 years (16,0 $\pm 0,11)$. Clinical, laboratory and instrumental methods for identification of endothelial dysfunction were used.

Results In the children with $\mathrm{AH}$ in association with endothelial dysfunction $(\mathrm{BMI}=16)$, metabolic changes and increased electrical activity of LV in ECG examination were revealed. The level of IVRT is elevated and indicates a decrease in the active ventricular relaxation $(109 \pm 9.41 \mathrm{~ms}$ versus $72.18 \pm$ 